\title{
Mitochondrial oxidative stress activates COX-2/mPGES-1/PGE2 cascade induced by albumin in renal proximal tubular cells
}

\author{
Yibo Zhuang ${ }^{1,2,3, *}$, Chenhu Wang ${ }^{1,2,3, *}$, Chunfeng Wu $\mathbf{u}^{1,2,3}$, Dan Ding ${ }^{1,2,3}$, Fei Zhao ${ }^{1,2,3}$, \\ Caiyu Hu ${ }^{1,2,3}$, Wei Gong ${ }^{1,2,3}$, Guixia Ding ${ }^{1,2,3}$, Yue Zhang ${ }^{1,2,3}$, Lihong Chen ${ }^{1,2,3}$, Guangrui \\ Yang $^{1,2,3}$, Chunhua Zhu ${ }^{1,2,3}$, Aihua Zhang ${ }^{1,2,3}$, Zhanjun Jia ${ }^{1,2,3}$ and Songming Huang ${ }^{1,2,3}$ \\ ${ }^{1}$ Department of Nephrology, Children's Hospital of Nanjing Medical University, Nanjing 210008, China \\ ${ }^{2}$ Jiangsu Key Laboratory of Pediatrics, Nanjing 210029, China \\ ${ }^{3}$ Nanjing Key Laboratory of Pediatrics, Nanjing 210008, China \\ *These authors equally contributed to this work \\ Correspondence to: Songming Huang, email: smhuang@njmu.edu.cn \\ Keywords: albumin; mitochondrial oxidative stress; COX-2; PGE2; proximal tubular cells \\ Received: November 19, $2017 \quad$ Accepted: January 04, $2018 \quad$ Published: January 12, 2018 \\ Copyright: Zhuang et al. This is an open-access article distributed under the terms of the Creative Commons Attribution License 3.0 \\ (CC BY 3.0), which permits unrestricted use, distribution, and reproduction in any medium, provided the original author and source \\ are credited.
}

\section{ABSTRACT}

COX-2/mPGES-1/PGE2 cascade is of importance in the pathogenesis of kidney injury. Meanwhile, recent studies documented a detrimental role of mitochondrial oxidative stress in kidney diseases. The present study was undertaken to investigate the role of mitochondrial oxidative stress in albumin-induced activation of COX-2/ mPGES-1/PGE2 cascade in renal proximal tubular cells. Following albumin overload in mice, we observed a significant increase of oxidative stress and mitochondrial abnormality determined by transmission electron microscope, which was attenuated by the administration of MnTBAP, a mitochondrial SOD2 mimic. More interestingly, albumin overload-induced upregulation of COX-2 and mPGES-1 at mRNA and protein levels was largely abolished by MnTBAP treatment in mice. Meanwhile, urinary PGE2 excretion was also blocked by MnTBAP treatment. Furthermore, mouse proximal tubule epithelial cells (mPTCs) were treated with albumin. Similarly, COX-2/mPGES-1/ PGE2 cascade was significantly activated by albumin in dose- and time-dependent manners, which was abolished by MnTBAP treatment in parallel with a blockade of oxidative stress. Collectively, the findings from current study demonstrated that mitochondrial oxidative stress could activate COX-2/mPGES-1/PGE2 cascade in proximal tubular cells under the proteinuria condition. Mitochondrial oxidative stress/ COX-2/mPGES-1/PGE2 could serve as the important targets for the treatment of proteinuria-associated kidney injury.

\section{INTRODUCTION}

Proteinuria plays an established role in mediating renal tubular injury and is viewed as a causative factor in promoting the progression of kidney diseases $[1,2]$. Evidence from numerous studies suggested that the cellular phenotypic changes and apoptotic response may serve as the underlying mechanisms of proteinuriainduced kidney injury [3, 4]. However, detailed molecular mechanisms remain elusive.
PGE2 is generated through a cyclooxygenases (COXs)/PGE2 synthases (PGESs) cascade [5]. To date, three PGE2 synthases including mPGES-1, mPGES-2, and cPGES were cloned with the best characterization of mPGES-1. The genetic deletion of mPGES-2 and cPGES did not reduce PGE2 production under basal or stress conditions $[6,7]$. Kidney is a major source of prostaglandins (PGs) including PGE2, PGD2, PGI2, PGF2 $\alpha$, and thromboxane A2 (TXA2). PGE2 is of importance in fluid metabolism regulation [8-11] and 
kidney injuries [12-14]. PGI2 and TXA2 are documented to be important in modulating renal hemodynamics $[15,16]$. The roles of PGD2 and PGF2 $\alpha$ in kidney remain uncertain. Our previous reports demonstrated that COX2 and mPGES-1 played detrimental role in CKD model of 5/6 nephrectomy [17] and AKI model of cisplatin nephropathy [18]. In diabetic kidney disease, blockade of COX-2 or PGE2 receptors of EP1 and EP4 resulted in significant protection against diabetic kidney injury [19]. In another proteinuric animal model induced by adriamycin, the overexpression of $\mathrm{COX}-2$ in podocytes remarkably worsened kidney damage [20]. All of these data suggest a critical role of the COX-2/mPGES-1/PGE2 cascade in mediating kidney injury.

Mitochondrial abnormality has been found in albumin-treated human proximal tubule cells [21] and CKDs [22, 23]. Moreover, our previous studies also demonstrated that mitochondrial oxidative stress contributes to the kidney injury induced by the aldosterone $[24,25]$. These findings highly suggested a detrimental role of mitochondrial dysfunction in proteinuric kidney diseases. Thus, in the present study, we investigated the role of mitochondrial oxidative stress in albuminuria-stimulated activation of COX-2/mPGES-1/PGE2 cascade in vivo and in vitro.

\section{RESULTS}

\section{Albumin overload-induced mitochondrial abnormality and oxidative stress was ameliorated by MnTBAP}

Eleven days of albumin overload in mice resulted in a severe structural disruption of the mitochondria in renal tubular cells as determined by TEM (Figure 1A). Meanwhile, the oxidative stress marker of MDA in urine was elevated by albumin overload (Figure 1B). Strikingly, all these abnormalities were reversed by a mitochondrial SOD2 mimic MnTBAP (Figure 1A and 1B).

\section{Activation of COX-2/mPGES-1/PGE2 cascade by albumin overload was blocked by MnTBAP in mice}

By qRT-PCR, we observed a selective upregulation of COX-2 and mPGES- 1 but not COX-1, mPGES-2, and cPGES (Figure 2A-2E). By Western blotting, we further confirmed the up-regulation of COX-2 and mPGES- 1 at the protein levels (Figure 3A-3C). Moreover, albumin overload significantly increased urinary PGE2 excretion (Figure 3D). Interestingly, application of MnTBAP largely normalized the stimulation of COX-2/mPGE-1/PGE2 cascade (Figure 3A-3D) without the impact on other PGE2 synthetic enzymes (Figure 2B-2D). These data suggested that albumin overload-induced oxidative stress might serve as a key factor leading to the stimulation of COX-2/mPGES-1/PGE2 cascade.

\section{Albumin directly stimulated COX-2/mPGES-1/ PGE2 cascade in renal proximal tubular cells}

To investigate whether albumin could directly stimulate COX-2/mPGES-1/PGE2 cascade in vitro, the mPTCs were treated with albumin, and the regulation of PGE2 synthetic enzymes including COX-2, COX-1, mPGES-1, mPGES-2, and cPGES was determined by qRT-PCR and Western blotting. As shown by the data, albumin dose-dependently induced COX-2, mPGES-1 mRNA (Figure 4A-4E) and protein (Figure 5A-5C) expressions without affecting the other PGE2 synthetic enzymes. Meanwhile, a time-dependent stimulation of this COX-2/mPGES-1 pathway was also observed at mRNA (Figure 6A-6E) and protein (Figure 7A-7C) levels. In line with the stimulation of the COX-2/mPGES- 1 axis, the PGE2 release in the cell culture medium was also doseand time-dependently elevated (Figure 5D and Figure7D). All of these data indicated a direct effect of albumin on the stimulation of COX-2/mPGES-1/PGE2 cascade in renal proximal tubular cells.

\section{Inhibition of mitochondrial oxidative stress abolished albumin-induced activation of the COX-2/mPGES-1/PGE2 cascade in renal proximal tubular cells}

Following albumin treatment, the ROS production was significantly enhanced (Figure 8A-8B). After the administration of MnTBAP, the increments of COX-2, mPGES-1, and PGE2 were significantly reversed (Figure 9A-9E and Figure 10A-10D). However, mPGES-2, cPGES, and COX-1 were not affected by albumin or MnTBAP treatment (Figure 9B-9D). These results demonstrated that the albumin effect on the activation of COX-2/mPGES-1/PGE2 cascade was through a mitochondrial oxidative stress-mediated mechanism.

\section{DISCUSSION}

Proteinuria is a hallmark for the diagnosis of glomerular diseases. Growing evidence has also demonstrated that proteinuria is an independent causative factor mediating the progression of kidney diseases, possibly via the stimulation of the inflammatory response in tubular epithelial cells and the tubulointerstitial region. PGE2 is abundantly produced in the kidney and contributes to a number of physiological and pathological processes. Under pathological insults, PGE2 was mostly found to play a detrimental role in kidney diseases [12-14].

Treating renal epithelial cells or animals with albumin has been widely used to mimic the effect of proteinuria 
on kidneys. In animals, albumin overload results in the exposure of renal tubular cells to the excessive albumin filtered through the glomerular filtration barrier. Although this model cannot entirely mimic the disease status of patients with proteinuria in the clinic, it could be a suitable model for evaluating the effect of albuminuria on kidney injury because of its exclusion of non-proteinuria factors, such as diabetes, hypertension, and lipid disorders. In the present study, albumin overload for 11 days induced severe renal tubular injury accompanied by a significant increase of oxidative stress and mitochondrial morphological alteration. In line with the elevated oxidative stress, the urinary PGE2 excretion was significantly increased. After administration of a mitochondrial SOD2 mimic, the oxidative stress occurred in kidneys was robustly abolished. At the same time, the abnormality of the mitochondrial morphology in the tubular cells was strikingly improved. These results suggested that mitochondrial-derived oxidative stress strongly contributed to the albumin overload-induced mitochondrial injury and might be an important pathogenic factor in mediating proteinuriaassociated kidney injury in patients.

PGE2 is an important inflammatory mediator that is involved in many kidney diseases [12, 13, 26, 27]. Blockade of the PGE2 synthetic enzymes [28] or antagonism of the PGE2 receptors substantially protects against various insults, such as diabetes, cisplatin nephropathy, adriamycin nephropathy and renal mass reduction [17-20]. Following the administration of albumin, urinary PGE2 was significantly elevated in line with the upregulation of COX-2 and mPGES-1 in kidneys. However, COX-1, mPGES-2 and cPGES were unaffected by the albumin overload. These data indicated that the activation of COX-2 and mPGES-1 might contribute to the renal PGE2 production in this model. This stimulation of COX-2/mPGES-1/PGE2 cascade was accompanied with increased oxidative stress and mitochondrial abnormality. Thus, we treated the animals with MnTBAP to evaluate the potential relationship between the activation of COX2/mPGES-1/PGE2 cascade and mitochondrial oxidative stress. Strikingly, MnTBAP almost entirely abolished the effect of albumin on the activation of this PGE2-generating cascade, suggesting a critical role of mitochondrial oxidative stress in this process. However, due to the complicated nature of mammalian systemic responses, we could not conclude whether these effects were directly from albumin exposure or a secondary response from kidney injury. To address this, we directly exposed mouse proximal tubular cells to the albumin and reproduced the phenomenon observed in vivo. As shown by the data, following MnTBAP treatment, the stimulation of the COX2/mPGES-1/PGE2 cascade was diminished in line with the

A
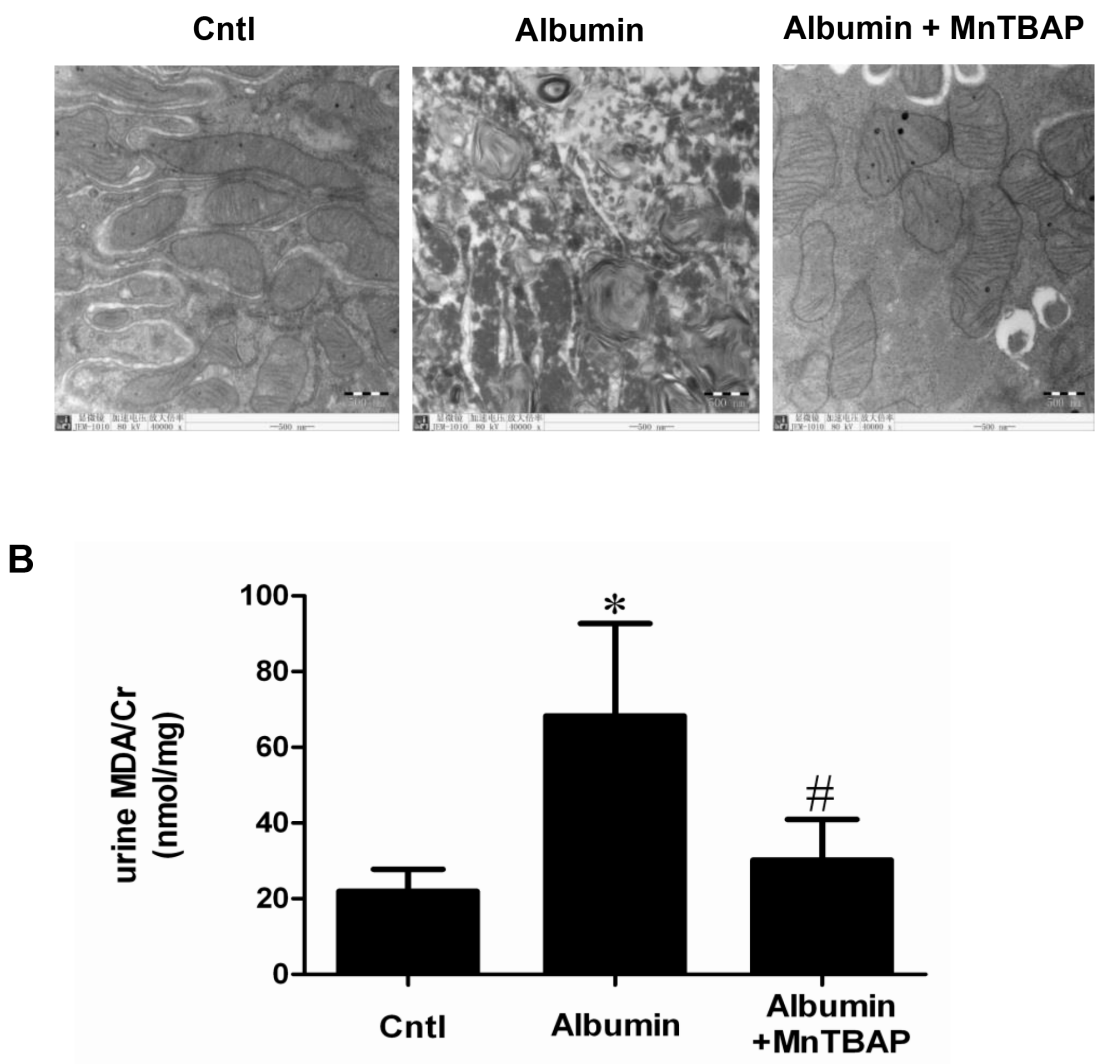

Figure 1: MnTBAP treatment ameliorated oxidative stress and mitochondrial abnormality induced by albumin overload in mice. (A) Mitochondrial morphology in tubular epithelial cells determined by TEM. (B) Renal TBARs levels. The values represent means $\pm \mathrm{SD}(n=8) .{ }^{*} P<0.01$ vs. control. ${ }^{\#} P<0.01$ vs. albumin-overload mice. 
attenuation of oxidative stress. These in vitro data clearly demonstrate a direct action of albumin on the stimulation of the COX-2/mPGES-1/PGE2 cascade via mitochondrial oxidative stress.

In summary, using both animals and in vitro cells, we demonstrated that albumin enhanced mitochondrial oxidative stress to stimulate COX-2/mPGES-1/PGE2 cascade in renal proximal tubular cells. These findings suggested that the inhibition of the mitochondrial oxidative stress could protect kidneys against proteinuriaassociated kidney injury, and such a protective effect might be through the inactivation of COX-2/mPGES-1/ PGE2 cascade to some extent.

\section{MATERIALS AND METHODS}

\section{Reagents and antibodies}

DMEM medium was purchased from Gibco Corporation (Carlsbad, CA, USA). Bovine serum

A
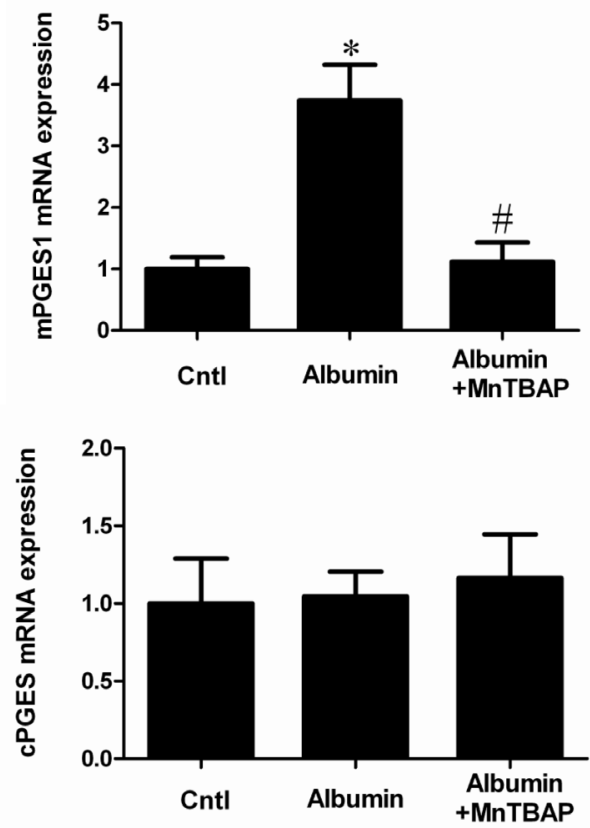

E

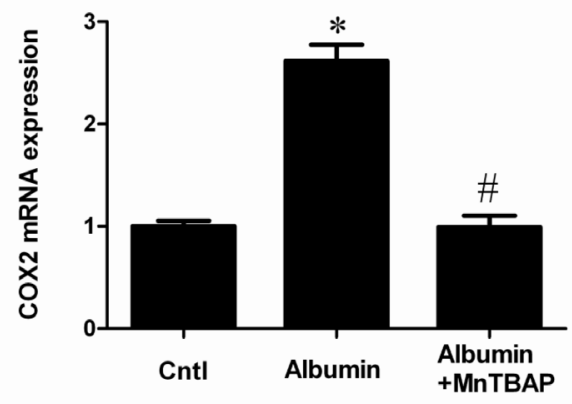

albumin (BSA) was obtained from Sigma Chemical Co. (St. Louis, MO, USA). MnTBAP was purchased from Sigma Chemical Co. (St. Louis, MO, USA). Rabbit polyclonal antibody against COX-2 and rabbit polyclonal antibody against mPGES-1 were purchased from Cayman Chemical (USA). Rabbit antibody against $\beta$-actin was obtained from Cell Signaling Technology (Danvers, MA, USA). Peroxidase-conjugated AffiniPure goat anti-rabbit secondary antibody was from Zhongshan Gold Bridge Biotechnology (Beijing, China).

\section{Animal studies}

For the MnTBAP experiment, 8-week-old 129/ Sv male mice weighing 25-30 g were subjected to an intraperitoneal (i.p.) injection with low-endotoxin albumin (A-9430, Sigma Chemical Co., St. Louis, MO) dissolved in saline for 11 days. In brief, albumin was administered for 5 days in a stepwise incremental dose regimen, increasing from $2 \mathrm{mg} / \mathrm{g}$ body weight on the first day (D1)

B

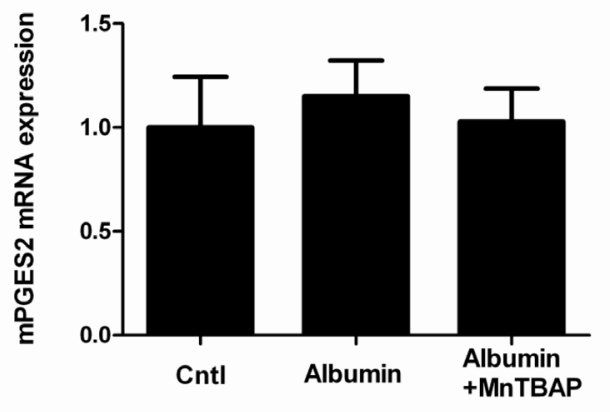

D

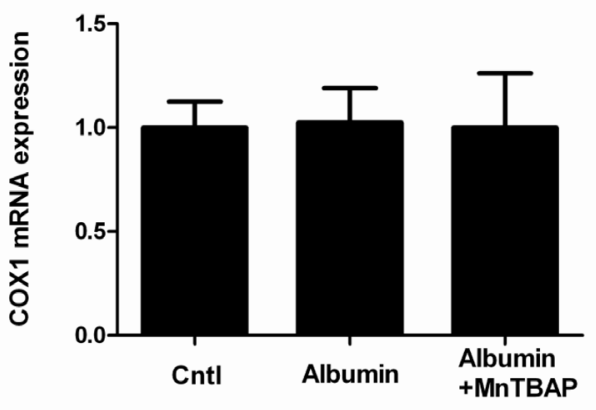

Figure 2: The upregulation of COX-2 and mPGES-1 was revered by MnTBAP at mRNA levels. (A) qRT-PCR analysis of mPGES-1. (B) qRT-PCR analysis of mPGES-2. (C) qRT-PCR analysis of cPGES. (D) qRT-PCR analysis of COX-1. (E) qRT-PCR analysis of COX-2. The values represent means $\pm \mathrm{SD}(n=8)$. ${ }^{*} P<0.01$ vs. control. ${ }^{\sharp} P<0.01$ vs. albumin-overload mice. 
to the maximum dose of $10 \mathrm{mg} / \mathrm{g}$ body weight on D5, which was thereafter maintained until day 11 . The control groups received a corresponding volume of saline via i.p. injection, and the albumin-treated mice received vehicle or MnTBAP (10 mg/kg/day) for 11 days.

All mice were maintained on a $12 \mathrm{~h}$ light-dark cycle in a temperature-controlled $\left(19-21^{\circ} \mathrm{C}\right)$ room, were fed a standard rodent diet, and were allowed free access to drinking water. At the termination of the experiments, the mice were anesthetized with an i.p. injection of a ketamine/xylazine/atropine cocktail. Plasma and kidney samples were then immediately frozen in liquid nitrogen and stored at $-80^{\circ} \mathrm{C}$ until use. The study protocols were reviewed and approved by the Institutional Animal Care and Use Committee at Nanjing Medical University, China.

\section{Cell culture studies}

mPTCs, an immortalized cell line, were grown in serum-free keratinocyte medium supplemented with bovine pituitary extract and epidermal growth factor (Wisent, Canada). The cells were specifically grown at $37^{\circ} \mathrm{C}$ with $5 \%$ $\mathrm{CO}_{2}$ and subcultured at $50-80 \%$ confluence using $0.25 \%$ trypsin- $0.02 \%$ EDTA (Invitrogen). In certain experiments, the cells were preincubated with MnTBAP (100 nmol/ml) for $30 \mathrm{~min}$ before BSA $(10 \mathrm{mg} / \mathrm{ml})$ treatment.

\section{Quantitative real-time PCR (qRT-PCR)}

Total RNA was extracted using the TRIzol reagent (Invitrogen),. Oligonucleotides were designed using Primer3 software (available at http://frodo.wi.mit. edu/) and synthesized by Invitrogen. The sequences of the primer pairs are shown in Table 1. qRT-PCR was then used to detect the mtDNA copy number and the mRNA expression of target genes. Reverse transcription was performed using a reaction kit (Promega Reverse Transcription System) according to the manufacturer's protocol. Real-time PCR amplification was performed using the ABI 7500 real-time PCR detection system (Foster City, CA, USA) with the SYBR Green PCR Master Mix (Applied Biosystems). The cycling conditions were $95^{\circ} \mathrm{C}$ for $10 \mathrm{~min}$, followed by 40 cycles of $95^{\circ} \mathrm{C}$ for $15 \mathrm{~s}$ and $60^{\circ} \mathrm{C}$ for $1 \mathrm{~min}$. The mRNA levels were normalized to GAPDH as a control and calculated using the comparative cycle threshold $(\Delta \Delta \mathrm{Ct})$ method.

\section{Western blotting}

mPTCs were lysed using a protein lysis buffer containing $50 \mathrm{mM}$ Tris, $150 \mathrm{mM} \mathrm{NaCl}, 10 \mathrm{mM}$ EDTA, $1 \%$ Triton X-100, $200 \mathrm{mM}$ sodium fluoride, and $4 \mathrm{mM}$ sodium orthovanadate as a protease inhibitor $(\mathrm{pH} 7.5)$. Immunoblotting was then performed with primary

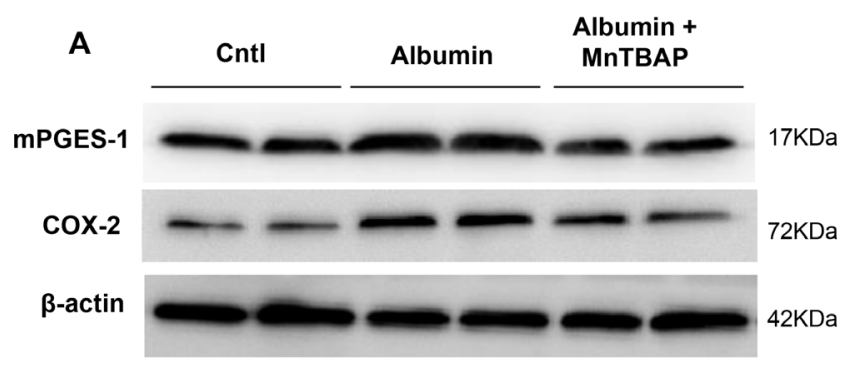

B

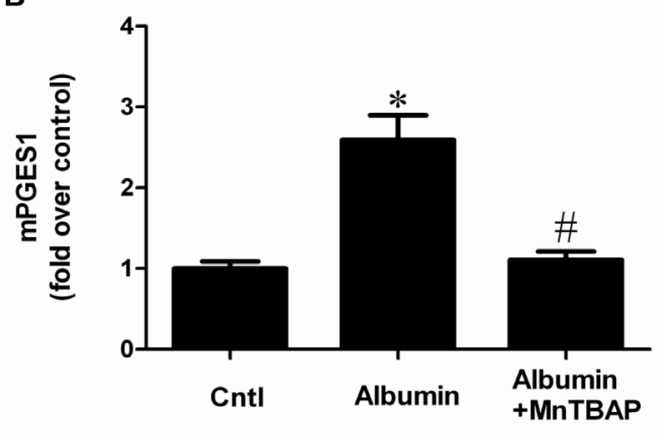

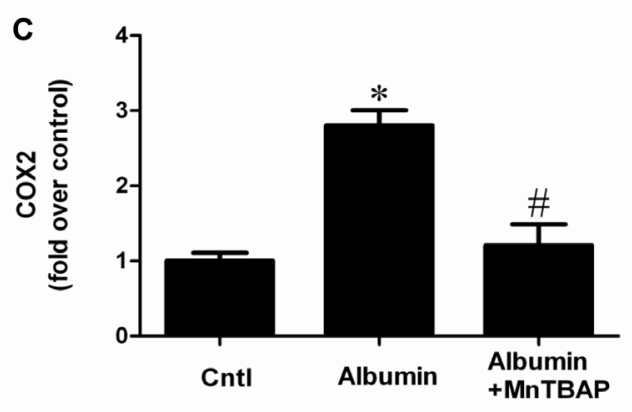

D

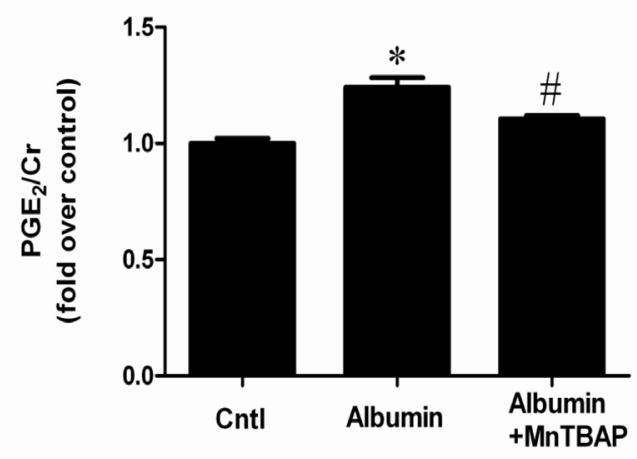

Figure 3: MnTBAP treatment reversed the effects of albumin overload on the protein expressions of COX-2 and mPGES-1 and urinary PGE2 production. (A) Western blots of mPGES-1, COX-2 and $\beta$-actin. (B) Densitometric analysis of mPGES-1. (C) Densitometric analysis of COX-2. (D) Urinary PGE2 output. The values represent means $\pm \operatorname{SD}(n=8) .{ }^{*} p<0.01$ vs. control. ${ }^{\#} p<0.01$ vs. albumin-overload mice. 
antibodies against COX-2 (Cayman Chemical, USA, 1:500), mPGES-1 (Cayman Chemical, USA, 1:500), and $\beta$-actin (1:1000), followed by the addition of HRP-labeled secondary antibodies. The blots were visualized using the Amersham ECL detection system (Amersham, Little Chalfont, UK). Densitometric analysis was performed using Quantity One software (Bio-Rad).

\section{EIA assay}

The concentration of PGE2 in the medium was examined using a commercial EIA kit purchased from Cayman Chemical.

\section{Analysis of ROS production in cells}

ROS production in mPTCs was measured by DCFDA as described previously [29].

A
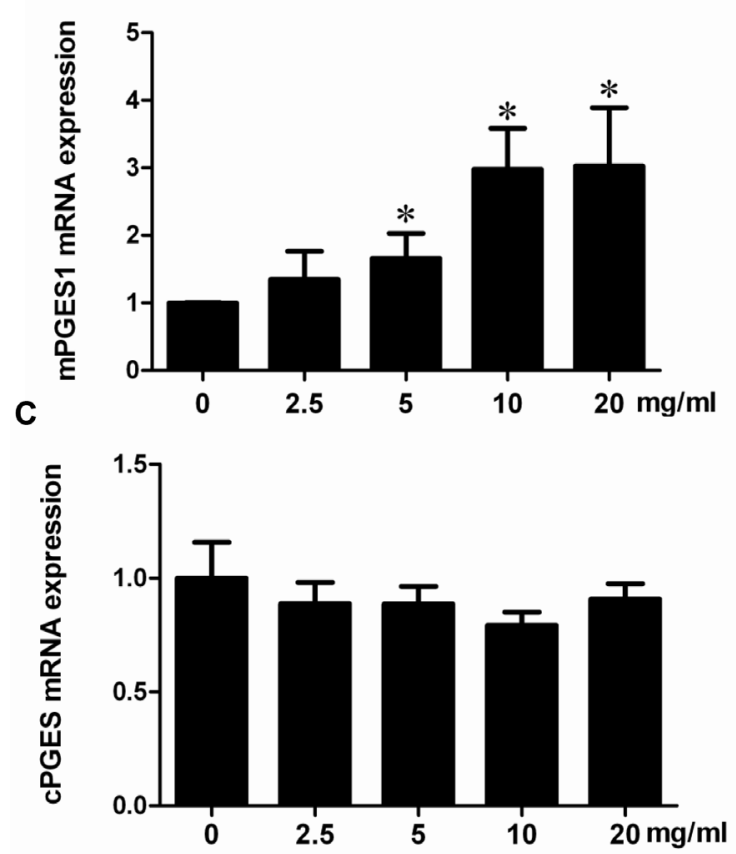

$E$

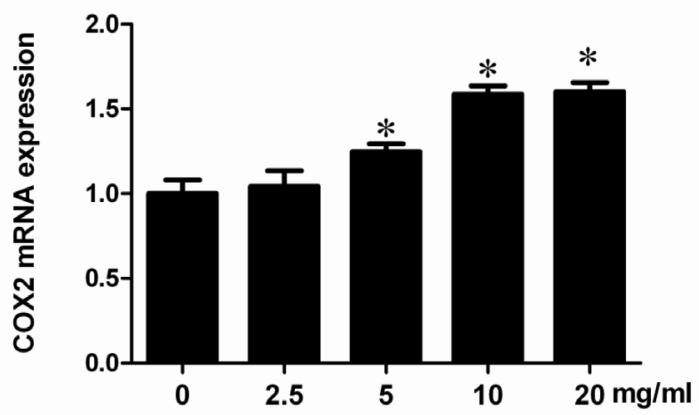

\section{Measurement of thiobarbituric acid-reactive} substances

The measurement of plasma thiobarbituric acidreactive substances (TBARS) was based on the formation of malondialdehyde by using a commercially available TBARS Assay kit (10009055; Cayman Chemical), according to the manufacturer's instructions.

\section{Transmission electron microscopy (TEM)}

Fresh kidney tissues were fixed in $1.25 \%$ glutaraldehyde/0.1 M phosphate buffer and post-fixed in $1 \%$ OsO4/0.1 M phosphate buffer. Ultrathin sections $(60 \mathrm{~nm})$ were then cut on a microtome, placed on copper grids, stained with uranyl acetate and lead citrate, and examined under an electron microscope (JEOL JEM-1010, Tokyo, Japan).

\section{B}
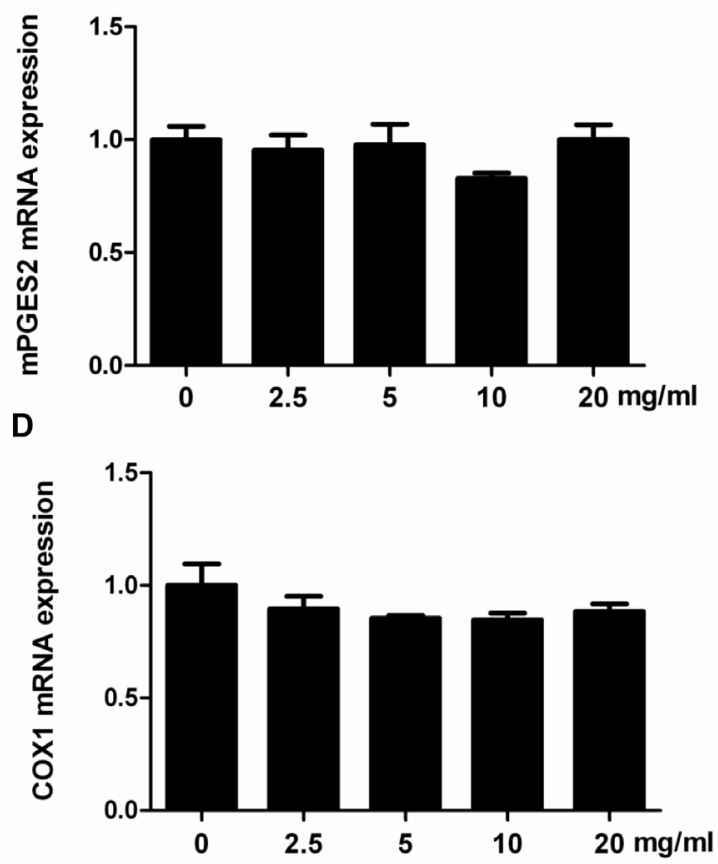

Figure 4: Albumin upregulated the mRNA expressions of COX-2 and mPGES-1 in a dose-dependent manner in mPTCs. (A) qRT-PCR analysis of mPGES-1. (B) qRT-PCR analysis of mPGES-2. (C) qRT- PCR analysis of cPGES. (D) qRT-PCR analysis of COX-1. (E) qRT-PCR analysis of COX-2. The values represent means $\pm \mathrm{SD}(n=6) .{ }^{*} P<0.01$ vs. control. 

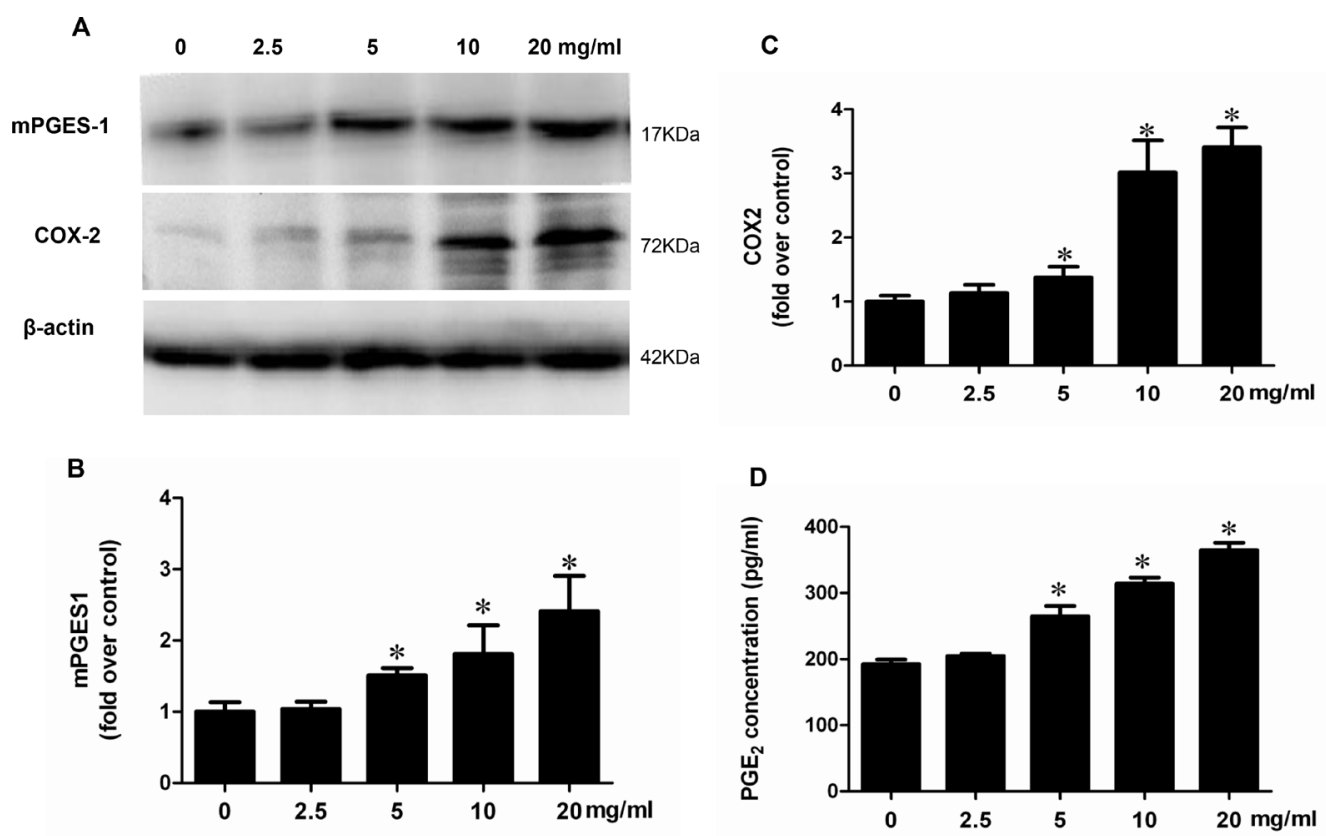

Figure 5: Albumin stimulated the protein expressions of COX-2 and mPGES-1 and PGE2 release in a dose-dependent manner in mPTCs. (A) Western blots of mPGES-1, COX-2, and $\beta$-actin. (B) Densitometric analysis of mPGES-1. (C) Densitometric analysis of COX-2. (D) PGE2 concentration in medium. The values represent means $\pm \operatorname{SD}(n=6)$.

A

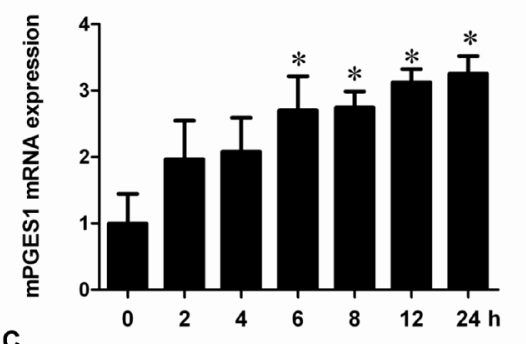

C

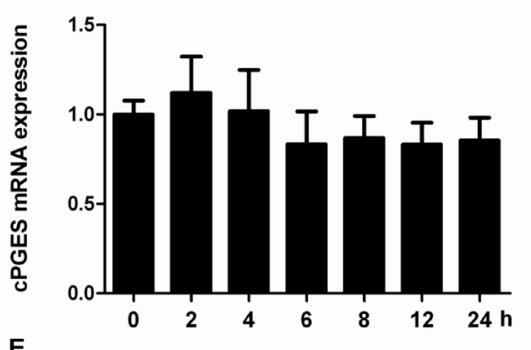

E

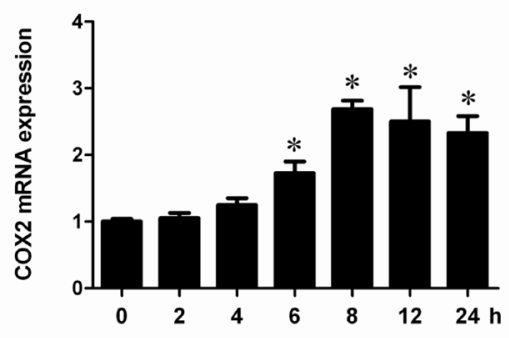

B

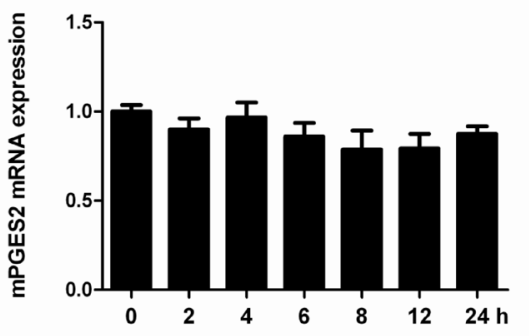

D

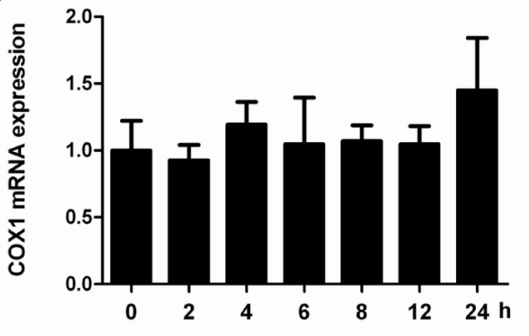

Figure 6: Albumin upregulated the mRNA expressions of COX-2 and mPGES-1 in a time-dependent manner in mPTCs. (A) qRT-PCR analysis of mPGES-1. (B) qRT-PCR analysis of mPGES-2. (C) qRT- PCR analysis of cPGES. (D) qRT-PCR analysis of COX-1. (E) qRT-PCR analysis of COX-2. The values represent means $\pm \operatorname{SD}(n=6) .{ }^{*} P<0.01$ vs. control. 
A

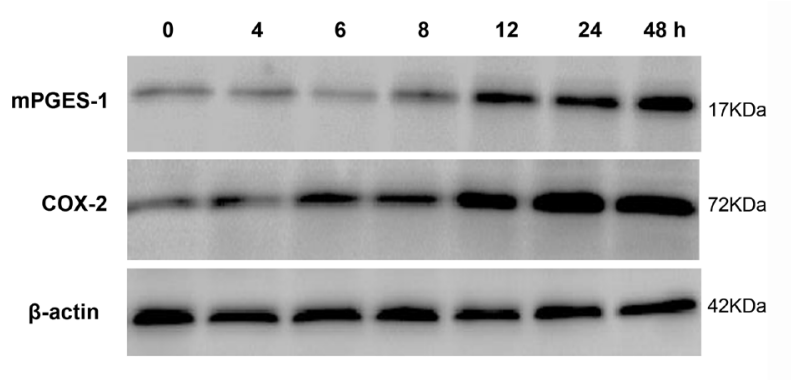

B

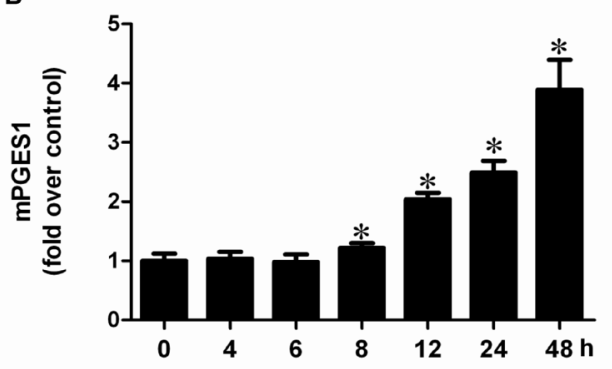

C

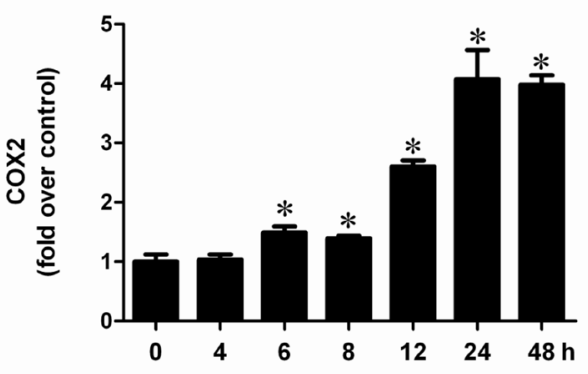

D

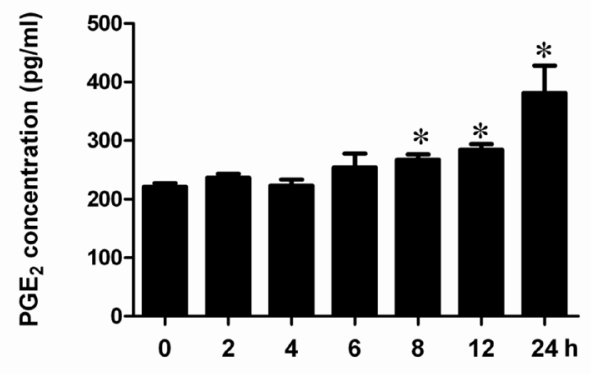

Figure 7: Albumin stimulated the protein expressions of COX-2 and mPGES-1 and PGE2 release in a time-dependent manner in mPTCs. (A) Western blots of mPGES-1, COX-2, and $\beta$-actin. (B) Densitometric analysis of mPGES-1. (C) Densitometric analysis of COX-2. (D) PGE2 concentration in medium. The values represent means $\pm \mathrm{SD}(n=6) .{ }^{*} P<0.01$ vs. control.

A
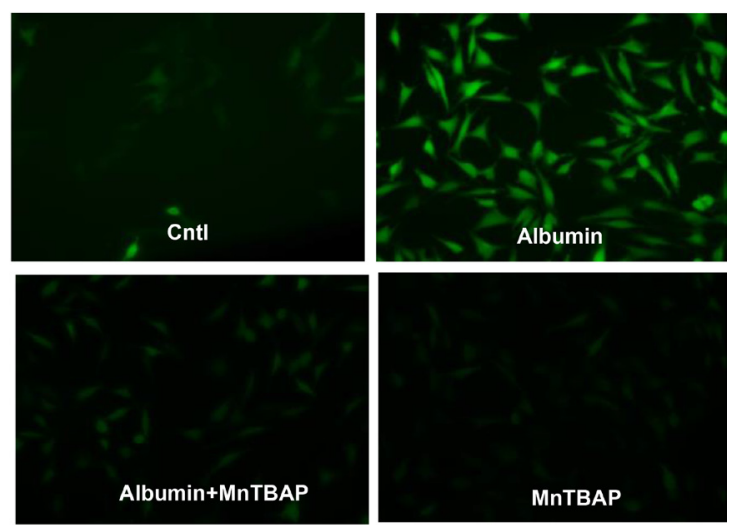

B

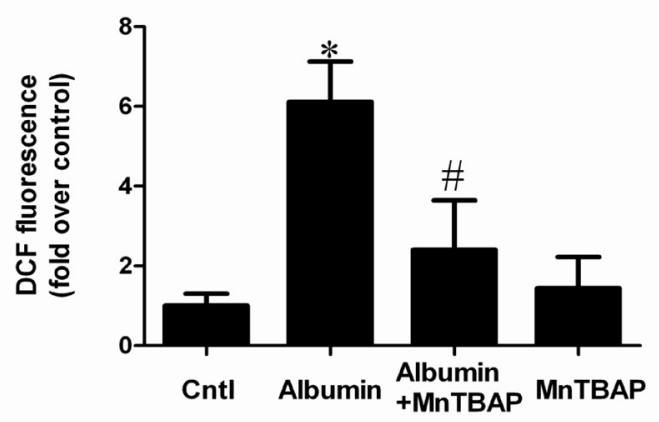

Figure 8: MnTBAP treatment suppressed albumin-induced ROS production in mPTCs. (A) Representative image of DCF fluorescence. (B) Quantitative analysis of ROS production by DCF fluorescence. The values represent means $\pm \operatorname{SD}(n=6)$. ${ }^{*} P<0.01$ vs. control. ${ }^{\#} P<0.01$ vs. albumin group. 
A

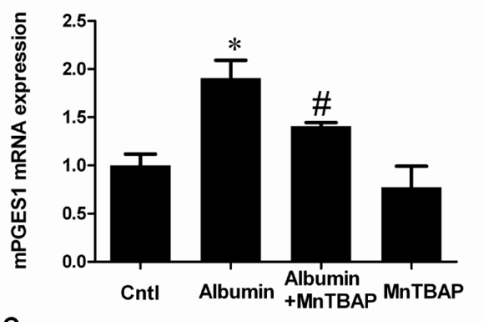

C

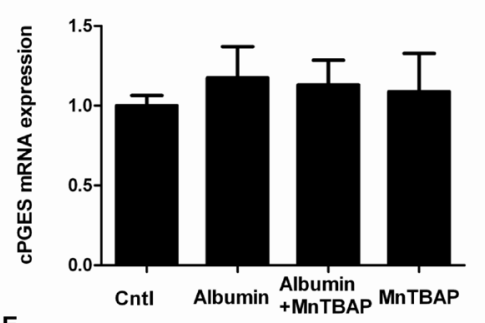

$\mathrm{E}$

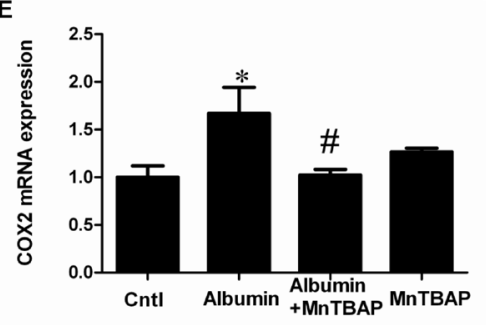

B

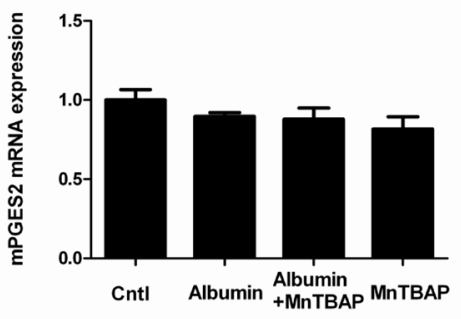

D

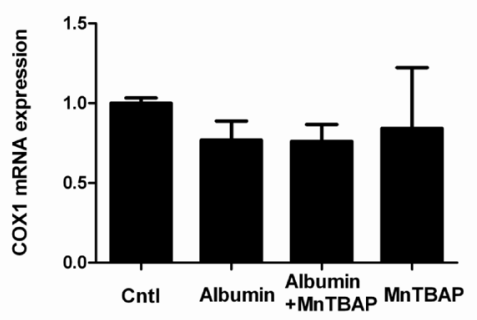

Figure 9: The upregulation of COX-2 and mPGES-1 mRNA expressions was revered by MnTBAP in mPTCs. (A) qRTPCR analysis of mPGES-1. (B) qRT-PCR analysis of mPGES-2. (C) qRT-PCR analysis of cPGES. (D) qRT-PCR analysis of COX-1. (E) qRT-PCR analysis of COX-2. The values represent means $\pm \mathrm{SD}(n=6) .{ }^{*} P<0.01$ vs. control. ${ }^{\#} P<0.01$ vs. albumin group.

A

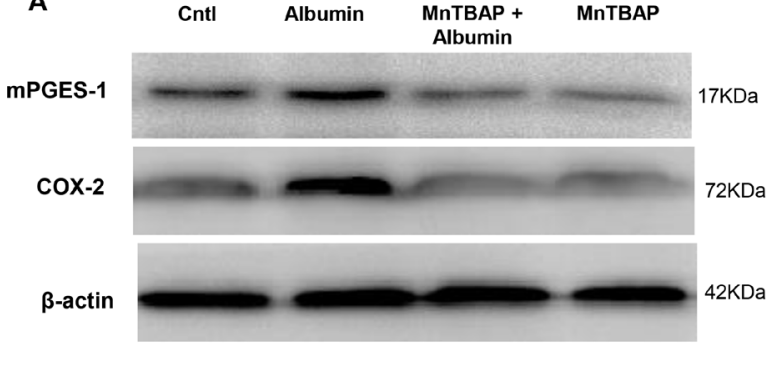

B

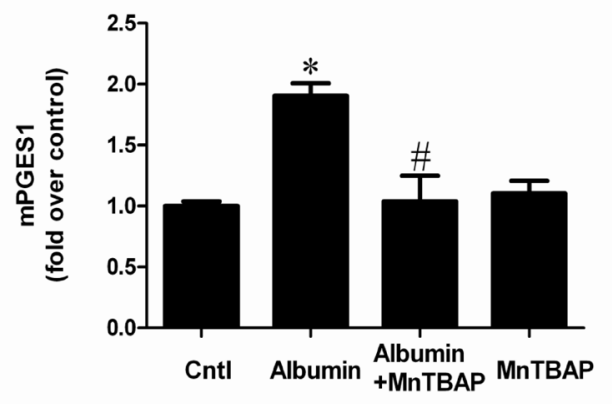

C

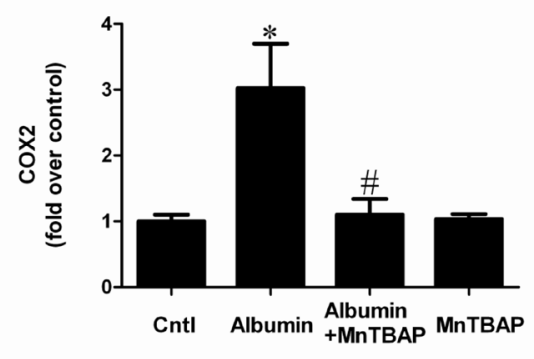

D

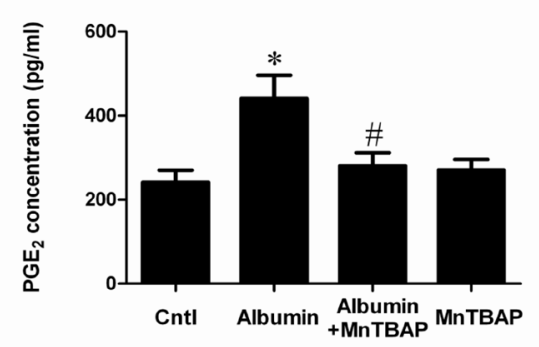

Figure 10: MnTBAP treatment reversed the effects of albumin on the protein expressions of COX-2 and mPGES-1 and PGE2 release in mPTCs. (A) Western blots of mPGES-1, COX-2 and $\beta$-actin. (B) Densitometric analysis of mPGES-1. (C) Densitometric analysis of COX-2. (D) PGE2 release in medium. The values represent means $\pm \operatorname{SD}(n=6)$. ${ }^{*} P<0.01$ vs. control. ${ }^{\#} P<0.01$ vs. albumin group. 


\begin{tabular}{ll}
\hline Gene symbol & Primer sequences \\
\hline GAPDH & 5'- GTCTTCACTACCATGGAGAAGG - 3' \\
mPGES1 & 5'- TCATGGATGACCTTGGCCAG -3' \\
& 5'- GGATGCGCTGAAACGTGGA - 3' \\
mPGES2 & 5'- CAGGAATGAGTACACGAAGCC - 3' \\
& 5'- CCTCGACTTCCACTCCCTG - 3' \\
cPGES & 5'- TGAGGGCACTAATGATGACAGAG - 3' \\
& 5'- TGTTTGCGAAAAGGAGAATCCG - 3' \\
COX1 & 5'- CCATGTGATCCATCATCTCAGAG - 3' \\
& 5'- ATGAGTCGAAGGAGTCTCTCG - 3' \\
COX2 & 5'- GCACGGATAGTAACAACAGGGA - 3' \\
& 5'- AACCGTGGGGAATGTATGAG - 3' \\
& 5'- GCAGGAAGGGGATGTTGTT - 3' \\
\hline
\end{tabular}

\section{Statistical analysis}

All data are presented as means \pm standard deviation (SD). The statistical analysis was performed using ANOVA followed by Bonferroni's test with SPSS 13 statistical software. $P<0.05$ was considered significant.

\section{Abbreviations}

mPTCs: mouse proximal tubule epithelial cells; CKD: chronic kidney disease; COX-1: cyclooxygenase-1; COX-2: cyclooxygenase-2; PGE2: prostaglandin E2; mPGES-1: membrane associated PGE synthase-1; mPGES-2: membrane associated PGE synthase-2; cPGES: cytosolic PGE2 synthase; BSA: bovine serum albumin; TEM: transmission electron microscopy; TBARS: thiobarbituric acid-reactive substances.

\section{Author contributions}

$\mathrm{SH}, \mathrm{AZ}$, and ZJ designed the study and experiments. $\mathrm{YZ}, \mathrm{CW}, \mathrm{CW}, \mathrm{DD}, \mathrm{FZ}, \mathrm{CH}, \mathrm{WG}, \mathrm{GD}, \mathrm{YZ}, \mathrm{LC}, \mathrm{GY}$, and $\mathrm{CZ}$ performed the research and analyzed the data. All authors interpreted and discussed the data. SH and ZJ wrote the manuscript. All authors read and approved the final manuscript.

\section{CONFLICTS OF INTEREST}

There is no conflicts of interest to disclose.

\section{FUNDING}

This work was supported by Grants from the National Natural Science Foundation of China (nos. 81370802, 81400750, 81300591, 81670647, 81600557,
81570643 and 81570616), the National Key Research and Development Program (no. 2016YFC0906103), the Natural Science Foundation of Jiangsu Province (no. BK20130077), and Nanjing City Key Medical Research Project (ZKX16057).

\section{REFERENCES}

1. Burton C, Harris KP. The role of proteinuria in the progression of chronic renal failure. Am J Kidney Dis. 1996; 27:765-775.

2. Eddy AA. Proteinuria and interstitial injury. Nephrol Dial Transplant. 2004; 19:277-281.

3. Zoja C, Morigi M, Remuzzi G. Proteinuria and phenotypic change of proximal tubular cells. J Am Soc Nephrol. 2003; 14:S36-41.

4. Li X, Pabla N, Wei Q, Dong G, Messing RO, Wang CY, Dong Z. PKC-delta promotes renal tubular cell apoptosis associated with proteinuria. J Am Soc Nephrol. 2010; 21:1115-1124.

5. Murakami M, Naraba H, Tanioka T, Semmyo N, Nakatani Y, Kojima F, Ikeda T, Fueki M, Ueno A, Oh S, Kudo I. Regulation of prostaglandin E2 biosynthesis by inducible membrane-associated prostaglandin E2 synthase that acts in concert with cyclooxygenase-2. J Biol Chem. 2000; 275:32783-32792.

6. Jania LA, Chandrasekharan S, Backlund MG, Foley NA, Snouwaert J, Wang IM, Clark P, Audoly LP, Koller BH. Microsomal prostaglandin E synthase-2 is not essential for in vivo prostaglandin E2 biosynthesis. Prostaglandins Other Lipid Mediat. 2009; 88:73-81.

7. Lovgren AK, Kovarova M, Koller BH. cPGES/p23 is required for glucocorticoid receptor function and embryonic growth but not prostaglandin E2 synthesis. Mol Cell Biol. 2007; 27:4416-4430. 
8. Stokes JB, Kokko JP. Inhibition of sodium transport by prostaglandin E2 across the isolated, perfused rabbit collecting tubule. J Clin Invest. 1977; 59:1099-1104.

9. Stokes JB. Effect of prostaglandin E2 on chloride transport across the rabbit thick ascending limb of Henle. Selective inhibitions of the medullary portion. J Clin Invest. 1979; 64:495-502.

10. Stokes JB. Integrated actions of renal medullary prostaglandins in the control of water excretion. Am J Physiol. 1981; 240:F471-480.

11. Guan Y, Zhang Y, Breyer RM, Fowler B, Davis L, Hebert RL, Breyer MD. Prostaglandin E2 inhibits renal collecting duct $\mathrm{Na}+$ absorption by activating the EP1 receptor. J Clin Invest. 1998; 102:194-201.

12. Mohamed R, Jayakumar C, Ranganathan PV, Ganapathy V, Ramesh G. Kidney proximal tubular epithelial-specific overexpression of netrin-1 suppresses inflammation and albuminuria through suppression of COX-2-mediated PGE2 production in streptozotocin-induced diabetic mice. Am J Pathol. 2012; 181:1991-2002.

13. Ranganathan PV, Jayakumar C, Mohamed R, Dong Z, Ramesh G. Netrin-1 regulates the inflammatory response of neutrophils and macrophages, and suppresses ischemic acute kidney injury by inhibiting COX-2-mediated PGE2 production. Kidney Int. 2013; 83:1087-1098.

14. Ren Y, D'Ambrosio MA, Garvin JL, Wang H, Carretero OA. Prostaglandin E2 mediates connecting tubule glomerular feedback. Hypertension. 2013; 62:1123-1128.

15. Fujita T, Fuke Y, Satomura A, Hidaka M, Ohsawa I, Endo M, Komatsu K, Ohi H. PG12 analogue mitigates the progression rate of renal dysfunction improving renal blood flow without glomerular hyperfiltration in patients with chronic renal insufficiency. Prostaglandins Leukot Ess ent Fatty Acids. 2001; 65:223-227.

16. Uriu K, Kaizu K, Hashimoto O, Komine N, Etoh S. Acute and chronic effects of thromboxane A2 inhibition on the renal hemodynamics in streptozotocin-induced diabetic rats. Kidney Int. 1994; 45:794-802.

17. Jia Z, Wang H, Yang T. Microsomal prostaglandin E synthase 1 deletion retards renal disease progression but exacerbates anemia in mice with renal mass reduction. Hypertension. 2012; 59:122-128.

18. Jia Z, Wang $N$, Aoyagi $T$, Wang $H$, Liu $H$, Yang $T$. Amelioration of cisplatin nephrotoxicity by genetic or pharmacologic blockade of prostaglandin synthesis. Kidney Int. 2011; 79:77-88.
19. Jia Z, Sun Y, Liu S, Liu Y, Yang T. COX-2 but Not mPGES-1 Contributes to Renal PGE2 Induction and Diabetic Proteinuria in Mice with Type-1 Diabetes. PLoS One. 2014; 9:e93182.

20. Cheng H, Wang S, Jo YI, Hao CM, Zhang M, Fan X, Kennedy C, Breyer MD, Moeckel GW, Harris RC. Overexpression of cyclooxygenase-2 predisposes to podocyte injury. J Am Soc Nephrol. 2007; 18:551-559.

21. Erkan E, Devarajan P, Schwartz GJ. Mitochondria are the major targets in albumin-induced apoptosis in proximal tubule cells. J Am Soc Nephrol. 2007; 18:1199-1208.

22. Granata S, Zaza G, Simone S, Villani G, Latorre D, Pontrelli P, Carella M, Schena FP, Grandaliano G, Pertosa G. Mitochondrial dysregulation and oxidative stress in patients with chronic kidney disease. BMC Genomics. 2009; 10:388.

23. Che R, Yuan Y, Huang S, Zhang A. Mitochondrial dysfunction in the pathophysiology of renal diseases. Am J Physiol Renal Physiol. 2014; 306:F367-378.

24. Yuan Y, Huang S, Wang W, Wang Y, Zhang P, Zhu C, Ding G, Liu B, Yang T, Zhang A. Activation of peroxisome proliferator-activated receptor-gamma coactivator 1alpha ameliorates mitochondrial dysfunction and protects podocytes from aldosterone-induced injury. Kidney Int. 2012; 82:771-789.

25. Wen H, Ting JP, O'Neill LA. A role for the NLRP3 inflammasome in metabolic diseases-did Warburg miss inflammation? Nat Immunol. 2012; 13:352-357.

26. Nakagawa N, Yuhki K, Kawabe J, Fujino T, Takahata O, Kabara M, Abe K, Kojima F, Kashiwagi H, Hasebe N, Kikuchi K, Sugimoto Y, Narumiya S, et al. The intrinsic prostaglandin E2-EP4 system of the renal tubular epithelium limits the development of tubulointerstitial fibrosis in mice. Kidney Int. 2012; 82:158-171.

27. Kvirkvelia N, McMenamin M, Chaudhary K, Bartoli M, Madaio MP. Prostaglandin E2 promotes cellular recovery from established nephrotoxic serum nephritis in mice, prosurvival, and regenerative effects on glomerular cells. Am J Physiol Renal Physiol. 2013; 304:F463-470.

28. Murakami M, Kudo I. Prostaglandin E synthase: a novel drug target for inflammation and cancer. Curr Pharm Des. 2006; 12:943-954.

29. Huang S, Zhang A, Ding G, Chen R. Aldosterone-induced mesangial cell proliferation is mediated by EGF receptor transactivation. Am J Physiol Renal Physiol. 2009; 296:F1323-1333. 